\title{
Cost-effectiveness analysis of empagliflozin in the treatment of patients with type 2 diabetes and established cardiovascular disease in Italy, based on the results of the EMPA-REG OUTCOME study
}

\author{
Sergio lannazzo ${ }^{1}$, Edoardo Mannucci ${ }^{2}$, Odette Reifsnider ${ }^{3}$, Aldo Pietro Maggioni ${ }^{4}$ \\ ${ }^{1}$ SIHS Health Economics Consulting, Turin, Italy \\ ${ }^{2}$ University of Florence, Florence, Italy \\ ${ }^{3}$ Evidera Inc., Bethesda, MD, USA \\ ${ }^{4}$ ANMCO (National Association of Hospital Cardiologists) Research Center, Florence, Italy
}

\begin{abstract}
INTRODUCTION: The EMPA-REG OUTCOME trial demonstrated the efficacy of empagliflozin in the treatment of type 2 diabetes (T2D) with a previous history of cardiovascular (CV) disease. The drug is currently reimbursed for T2D Italian patients, but the reduction of CV mortality and morbidity shown in the trial opens a new treatment perspective in those patients with associated high $\mathrm{CV}$ risk.

OBJECTIVE: Cost-effectiveness analysis of empagliflozin for the treatment of T2D patients with a previous history of CV disease, from the Italian National Health Service (NHS) perspective.

METHODS: The analysis was performed with an individual simulation model, which can predict the time to CV events or death through a set of time-dependent regressions estimated on the patient-level data of the EMPA-REG OUTCOME trial. This design allows the direct simulation of long-term outcomes and costs without the need for surrogate endpoints. The model was adapted to the Italian setting, considering local epidemiological data, baseline quality of life (QoL) utility, background mortality and unit costs from current prices and tariffs. The cost perspective was that of the Italian NHS and the horizon of the simulation was lifetime. Costs and benefits were discounted at a $3.5 \%$ rate.

RESULTS: Base case results were estimated on a cohort of 5,000 patients, which ensured the convergence of the simulation. Patients treated with empagliflozin in add-on to the standard of care (SoC) lived on the average 13.8 undiscounted years as compared to 11.8 years of patients on SoC alone. The gain in discounted quality-adjusted life years (QALYs) was 1.0, due to improved survival and QoL linked to the reduced incidence of CV events and CV mortality. The incremental cost-effectiveness ratio (ICER) was 4,811 €/QALY, well below the commonly applied threshold of 30,000-50,000€/QALY. CONCLUSION: Empagliflozin in add-on to the SoC is a highly cost-effective strategy for the treatment of T2D patients with known CV disease in the Italian setting.
\end{abstract}

\section{Keywords}

Cost-effectiveness; Cost-utility; Type 2 diabetes; Cardiovascular risk; Empagliflozin; EMPA-REG OUTCOME

\section{INTRODUCTION AND OBJECTIVE}

Type 2 diabetes (T2D) - the most common form of diabetes, with a prevalence of about $90 \%$ of cases - is identified as an important risk factor for cardiovascular events [1,2] Cardiovascular diseases are also the leading cause of death in diabetic subjects worldwide, amounting to about $50 \%$ in T2D patients [3].
The clinical trial EMPA-REG OUTCOME investigated the efficacy of empagliflozin $-\mathrm{a}$ specific and reversible inhibitor of the sodium-glucose co-transporter type 2 (SGLT2) - in reducing the incidence of cardiovascular events and mortality in a cohort of 7,020 patients with T2D and a previous history of established cardiovascular disease [4,5].
Corresponding author Sergio lannazzo sergio.iannazzo@icloud.com

\section{Disclosure}

This analysis was funded by Boehringer Ingelheim Italia. 
Patients were randomized to receive empagliflozin or placebo in addition to their standard therapy. The primary endpoint of the study - defined as a composite endpoint of death from cardiovascular causes, (nonfatal) myocardial infarction or stroke - was achieved over a median follow-up of 3.1 years, with an incidence of $10.5 \%$ in the empagliflozin group and $12.1 \%$ in the placebo group (hazard ratio $=0.86$; CI95\%: $0.74-$ $0.99 ; \mathrm{p}=0.04$ for superiority). Among the secondary endpoints, cardiovascular mortality was reduced by $38 \%$, mortality for any cause was reduced by $32 \%$, hospitalizations for heart failure were reduced by $35 \%$, and kidney damage progression was reduced by $39 \%[4,5]$.

Empagliflozin is currently authorized and reimbursed by the Italian National Health Service (NHS) for use in T2D patients; however, the clinical evidence derived from the EMPA-REG OUTCOME study opens a new treatment perspective in the patient at high risk due to established cardiovascular disease. In this sense, it is important to explore its effects and consequences also in the economic context. In fact, a comprehensive assessment of a health technology - besides the evaluation of its clinical efficacy and safety - should also include an assessment of its economic effectiveness and sustainability, with the aim of satisfying the need to rationalize the allocation and use of the available resources, by providing the healthcare decision-maker with objective evaluation criteria. In general, comprehensive economic evaluations consist of two elements. The first type of analysis - usually carried out in the form of cost-effectiveness, or cost-utility, over a medium to long-term time horizon - aims at estimating the efficiency of the allocation of health resources for a certain technology. The second type, typically defined as a budget impact analysis, answers the most immediate and frequent question of the decision maker about short-term (1-3 years) financial sustainability.

The economic assessment of empagliflozin in diabetic patients with established cardiovascular disease was first addressed through an analysis of the second type [6]. The aim of this study is the cost-effectiveness evaluation of empagliflozin in addition to the standard of care therapy as compared to the standard of care alone in the perspective of the Italian NHS.

\section{MATERIALS AND METHODS}

The cost-effectiveness analysis was conducted in MS Excel, with the support of a simu- lation model, to directly integrate the statistical analyses developed on the patient data of the EMPA-REG OUTCOME study and to perform the pharmacoeconomic comparison between empagliflozin and the Standard of Care (SoC) therapy in subjects with T2D and established cardiovascular disease $[7,8]$. As detailed in the following paragraphs, the model allows to extrapolate the risk functions of cardiovascular and renal events directly from the EMPA-REG OUTCOME data, without the need to refer to surrogate outcomes. The economic model was adapted in order to carry out an analysis which is adherent with the Italian clinical setting. The cost-effectiveness analysis was conducted in the perspective of the Italian NHS, with a time horizon corresponding to the entire life of patients (lifetime horizon). Costs and benefits were discounted at the annual rate of $3.5 \%$.

\section{Model design}

The model is based on the patient-level simulation of the time to the occurrence of each of the nine possible cardiovascular or renal events, defined in accordance with the EMPA-REG OUTCOME study protocol [4,9]:

- Non-fatal myocardial infarction;

- Non-fatal ischemic stroke;

- Hospitalization due to heart failure;

- Transient ischemic attack;

- Coronary revascularization;

- Death from cardiovascular causes;

- New onset of macroalbuminuria;

- Kidney injury (doubling of serum creatinine level and eGFR $=45 \mathrm{ml} / \mathrm{min} / 1.73 \mathrm{~m}^{2}$ );

- The beginning of renal-replacement therapy.

A set of parametric functions embedded in the model - derived on the basis of multivariate and time-dependent regressions of patient data from the EMPA-REG OUTCOME study - allows to estimate the time to events, according to baseline characteristics and the occurrence of previous events. More details on the derivation of the parametric functions is reported in the Appendix A. The simulation flow is schematically represented in Figure 1. Each simulated patient is created based on the distribution of baseline characteristics and sent to the two comparator's arms, i.e. treatment with empagliflozin $+\mathrm{SoC}$ and SoC alone. At the beginning of the simulation, the time to the first event is determined. After this event, the quantities representing the outcomes (survival, quality-adjusted life years -QALY, and costs) are updated. In addition, risk functions are updated to take into account the event occurred. At this point, the simulation goes to the next event and pro- 


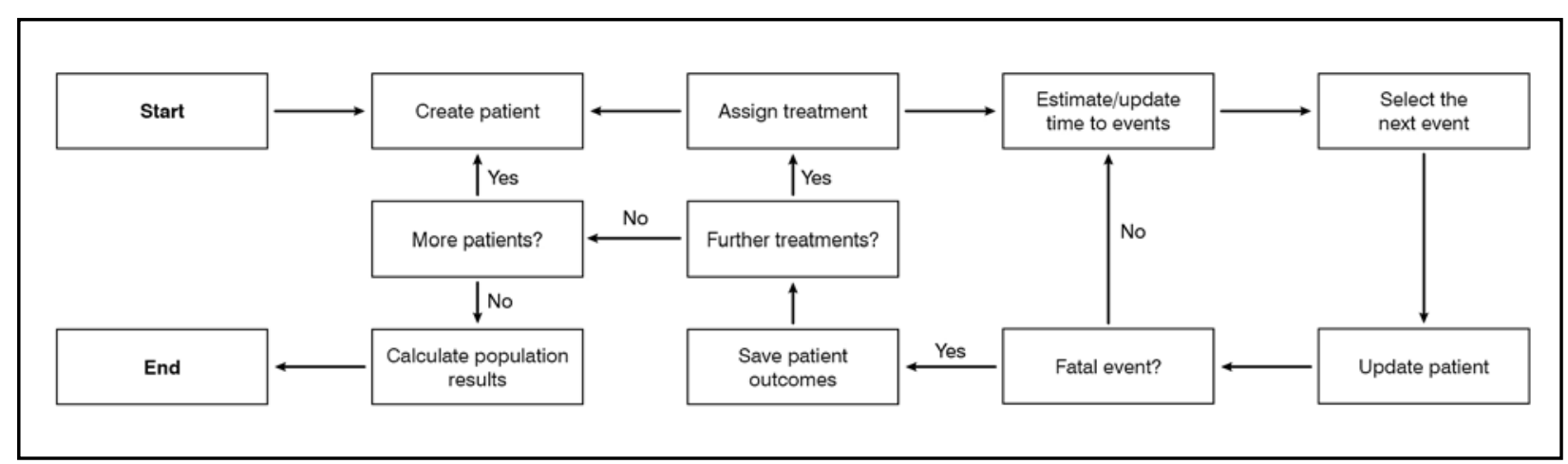

Figure 1. Patient-level simulation flow

\begin{tabular}{|c|c|c|c|c|}
\hline & $\begin{array}{l}\text { EMPA-REG OUTCOME } \\
\text { (Europe) }\end{array}$ & Italian data & $\begin{array}{c}\text { Population } \\
\text { (Italian data) }\end{array}$ & Source \\
\hline Age (mean $\pm \mathrm{SD})$ & $63.9 \pm 8.3$ & $66.0 \pm 9.0$ & T2D & [11] \\
\hline Female (\%) & 28.5 & 42.5 & $\mathrm{~T} 2 \mathrm{D}+\mathrm{CV}$ & [11] \\
\hline $\mathrm{BMI} \geq 30(\%)$ & 60.8 & 63.1 & T2D & [11] \\
\hline $\mathrm{HbA} 1 \mathrm{c} \geq 8.5 \%(\%)$ & 31.6 & 23.7 & T2D & {$[11]$} \\
\hline \multicolumn{5}{|l|}{ CV risk variables (\%) } \\
\hline BCV1 (previous stroke) & 27.1 & 15.5 & $\mathrm{~T} 2 \mathrm{D}+\mathrm{CV}$ & {$[11]$} \\
\hline BCV2 (MI) & 50.6 & 38.1 & $\mathrm{~T} 2 \mathrm{D}+\mathrm{CV}$ & {$[11]$} \\
\hline BCV3 (CABG) & 20.4 & $\mathrm{n} / \mathrm{a}$ & & \\
\hline BCV4 (MCAD) & 40.6 & $\mathrm{n} / \mathrm{a}$ & & \\
\hline BCV5 (SVCAD) & 11.8 & $\mathrm{n} / \mathrm{a}$ & & \\
\hline BCV 6 (PAD) & 23.4 & $n / a$ & & \\
\hline \multicolumn{5}{|c|}{ Renal risk variables (\%) } \\
\hline eGFR 60-90 & 53.4 & $\mathrm{n} / \mathrm{a}$ & & \\
\hline eGFR $<60$ & 21.8 & 23.8 & $\mathrm{~T} 2 \mathrm{D}$ & [12] \\
\hline
\end{tabular}

Table I. Baseline characteristics of the simulated population. Where available Italian epidemiological data on the population of diabetic patients with established cardiovascular disease (T2D $+C V$ ) or on the total population of diabetic (T2D) subjects were considered. Where Italian data were unavailable, the data of the subpopulation of the EMPA-REG OUTCOME study enrolled in Europe were considered

$\mathrm{CABG}=$ coronary artery bypass graft; $\mathrm{CAD}=$ coronary artery disease; $\mathrm{Ml}=$ myocardial infarction; $\mathrm{MCAD}=$ multi vessel $\mathrm{CAD} ; \mathrm{n} / \mathrm{a}=$ not available; $\mathrm{SVCAD}=$ single vessel CAD; PAD = peripheral arterial disease

ceeds in this way until the final event, represented by the death of the patient, after which the outcomes in terms of survival, QALY and cost are stored in the system. The risk of death is calculated for both cardiovascular causes (based on the EMPA-REG OUTCOME data) and for all other causes, using the mortality tables of the Italian population [10]. The simulation is repeated for a large number of iterations, determined in such a way as to allow for the convergence of the system. This condition occurs for a number of iterations, beyond which the median values of the simulation results remain substantially stable. When all patients have been simulated in both treatment arms, the overall outcomes are calculated, in order to estimate the cost-effectiveness of empagliflozin compared with SoC in subjects with T2D and established cardiovascular disease.

\section{Simulated population}

As described above, patients are individually simulated, with baseline characteristics describing the risk profile randomly extracted from statistical distributions. During the process of adaptation of the model to the Italian clinical setting a series of targeted literature searches were conducted to identify all relevant sources of input data. The baseline characteristics of the cohort of patients enrolled in the EMPA-REG OUTCOME study were replaced with Italian epidemiological data, available in the literature on the population of diabetic patients with established cardiovascular disease (T2D + CV) or, as a second choice, on the total population of diabetic (T2D) subjects $[11,12]$. Where Italian data were unavailable, the data of the subpopulation of the EMPA-REG OUTCOME study enrolled in Europe were considered (Table I). 


\begin{tabular}{lcc}
\hline & Utility, mean (95\%Cl) & Sources \\
\hline Base case utility diabetic population & 0.874 & {$[13,14]$} \\
Utility decrease related to events & & \\
Non-fatal myocardial infarction & $-0.047(-0.057 ;-0.036)$ & {$[15]$} \\
Non-fatal stroke & $-0.060(-0.074 ;-0.046)$ & {$[15]$} \\
Heart failure & $-0.050(-0.064 ;-0.036)$ & {$[15]$} \\
Transient ischemic attack & $-0.070(-0.131 ;-0.008)$ & {$[15]$} \\
Coronary revascularization & -0.030 & {$[16]$} \\
Macroalbuminuria & $-0.038(-0.059 ;-0.016)$ & {$[15]$} \\
Kidney injury ${ }^{1}$ & $-0.038(-0.059 ;-0.016)$ & {$[15]$} \\
Kidney failure $^{2}$ & $-0.038(-0.059 ;-0.016)$ & {$[15]$} \\
Effect of multiple events (additive with respect to the application of \\
individual decreases) & & \\
2 events & 0.017 & {$[15]$} \\
3 events & 0.042 & {$[15]$} \\
4 events & 0.070 & {$[15]$} \\
5 or more events & 0.087 & {$[15,16]$} \\
\hline
\end{tabular}

Table II. Utility considered in the cost-effectiveness analysis

${ }^{1}$ Doubling of serum creatinine level and eGFR $=45 \mathrm{ml} / \mathrm{min} / 1.73 \mathrm{~m}^{2}$

${ }^{2}$ Beginning of renal-replacement therapy

\section{Quality of life data}

Input data on the quality of life of patients with T2D and established cardiovascular disease are used to weigh the simulated survival and obtain the estimate of the QALYs gained in each of the two treatment arms. The base case utility was determined starting from the reference standard for the Italian population [13], reduced by the specific utility decrease related to the chronic diabetic condition (UK data in the absence of available Italian data) [14]. Specific utility reductions were also considered for all cardiovascular and renal events (Table II). These were obtained from a British study that estimated them based on a cohort of diabetic patients $[15,16]$.

\section{Cost data}

The cost-effectiveness analysis was carried out in the perspective of the Italian NHS, therefore only direct health costs were taken into account, with costing year 2017. More specifically, the costs related to drug acquisition and the management of cardiovascular and renal events were considered. Costs for drug acquisition were assessed considering the mix of drugs that define the standard treatment in subjects with T2D and established cardiovascular disease. This was estimated through the QuintilesIMS Patient Analyzer service, based on the quarterly collection of anonymous patient records among a panel of 140 diabetologists [17].

The daily cost of the individual drugs was calculated by considering the recommended daily dose, based on the respective summary of product characteristics (SPC), and the exfactory price or that from the transparency list for equivalent products $[18,19]$. In the case of treatments available on the market in various packages, the unit price was calculated by weighing the cost of the individual products according to their relevant market shares [20]. In order to calculate the daily cost of insulin in monotherapy or in combination with oral antidiabetic agents, an average requirement of $20 \mathrm{IU} /$ day for basal insulin and $50 \mathrm{IU} /$ day for basal-bolus regimens was assumed. The use of basal insulin was estimated at $100 \%$ in the case of combinations with sulphonylureas, GLP-1 and DPP-4, at $75 \%$ when associated with repaglinide, and at $38 \%$ in combination with metformin [17]. Based on the assumptions hereby defined, the cost of the standard treatment was 1.20 $€ /$ day. In line with the EMPA-REG OUTCOME study design, it was assumed that the treatment with empagliflozin was added to the standard treatment, resulting in a total cost for the empagliflozin + SoC arm of $2.55 € /$ day. However, the assumption of empagliflozin add-on is conservative in the perspective of the economic evaluation. In fact, it seems unlikely that in the clinical practice empagliflozin could simply be added without a concomitant adjustment of the ongoing therapy for each patient. Consequently, in concomitance with the base case, an alternative scenario was considered in which, in line with the previous budget impact analysis [6], it was assumed that the adoption of empagliflozin occurred as a replacement of an oral antidiabetic agent, except for patients under treatment with metformin, insulin and insulin associated with metformin. In this scenario, the cost of the drug in the empagliflozin + SoC arm was $1.86 € /$ day.

Hospitalization costs for cardiovascular and renal events (Table III) were derived from the Italian Hospital Tariff System for acute diseases, weighed for the relative hospitalization frequencies $[21,22]$. For cardiovascular death, it was assumed that - on average - only half of the cases are hospitalized. The cost of renal replacement therapy was estimated at $€ 41,027$, corresponding to the cost of kidney dialysis per patient per year [23]. Finally, it was assumed that, for the management of macroalbuminuria and kidney injury, there is no need for hospitalization, but outpatient services are sufficient.

\section{Sensitivity analysis and scenarios}

Cost-effectiveness analysis results were estimated for the base case and for an exploratory scenario. The latter concerned the cost 


\begin{tabular}{|c|c|c|c|}
\hline Event & Cost $(€)$ & Source & Reference \\
\hline $\begin{array}{l}\text { Non-fatal myocardial } \\
\text { infarction }\end{array}$ & $3,897.89$ & $\begin{array}{l}\text { DRG } 121 \text { - CV disorders with acute MI, CV complications, discharged alive } \\
\text { DRG } 122 \text { - CV disorders with acute MI, without CV complications, discharged alive }\end{array}$ & {$[21,22]$} \\
\hline Non-fatal stroke & $6,073.00$ & DRG 559 - Acute ischemic stroke with use of thrombolytic agent & [21] \\
\hline Heart failure & $3,052.00$ & DRG 127 - Heart failure and shock & [21] \\
\hline $\begin{array}{l}\text { Transient ischemic } \\
\text { attack }\end{array}$ & $2,543.00$ & DRG 524 - Transient ischemic attack & [21] \\
\hline $\begin{array}{l}\text { Coronary } \\
\text { revascularization }\end{array}$ & $8,080.55$ & $\begin{array}{l}\text { DRG } 547 \text { - Coronary bypass with cardiac catheter with major CV diagnosis } \\
\text { DRG } 549 \text { - Coronary bypass without cardiac catheter with major CV diagnosis } \\
\text { DGR } 553 \text { - Other vascular procedures with CV complications with major CV } \\
\text { diagnosis } \\
\text { DRG } 555 \text { - Percutaneous CV procedures with major CV diagnosis } \\
\text { DRG } 557 \text { - Percutaneous CV procedures with drug-eluting stent with major CV } \\
\text { diagnosis }\end{array}$ & {$[21,22]$} \\
\hline Macroalbuminuria & 22.26 & $\begin{array}{l}\text { 1 Creatinine test ( } 90.16 .4 \text { creatinine clearance) } \\
1 \text { Nephrological visit ( } 89.7 \text { GP visit) }\end{array}$ & {$[21]$} \\
\hline Kidney injury ${ }^{1}$ & 42.92 & $\begin{array}{l}\text { 1 Creatinine test (90.16.4 creatinine clearance) } \\
1 \text { Nephrological visit ( } 89.7 \text { GP visit) } \\
1 \text { Nutritional visit ( } 89.7 \text { GP visit) }\end{array}$ & [21] \\
\hline Kidney failure ${ }^{2}$ & $41,027.00$ & Annual cost for dialysis & [23] \\
\hline $\begin{array}{l}\text { Cardiovascular } \\
\text { death }\end{array}$ & $2,009.00$ & $\begin{array}{l}\text { DRG } 123 \text { - CV disorders with acute MI (assumption: } 50 \% \text { of sudden deaths/ not } \\
\text { hospitalized) }\end{array}$ & [21] \\
\hline
\end{tabular}

Table III. Hospitalization costs for cardiovascular and renal events

${ }^{1}$ Doubling of serum creatinine level and eGFR $=45 \mathrm{ml} / \mathrm{min} / 1.73 \mathrm{~m}^{2}$

${ }^{2}$ Beginning of renal-replacement therapy

$\mathrm{CV}=$ cardiovascular; $\mathrm{GP}=$ general practitioner; $\mathrm{MI}=$ myocardial infarction

of the drug, exploring the potential impact of considering empagliflozin in substitution for other antidiabetic drugs, and not simply as an add-on. In this scenario, the pharmaceutical cost in the empagliflozin + SoC arm was 1.86 $€ /$ day (as compared to $2.55 € /$ day in the base case).

In order to estimate the impact of the uncertainty of data on the results of the analysis, a probabilistic sensitivity analysis (PSA) was conducted, in which all the parameters of the model assume a probabilistic value distributed over their uncertainty range. The latter was assumed to be equal to CI95\%, where this was reported in the original sources. For derivative parameters or parameters for which uncertainty information was not available, a conventional standard deviation value of $\pm 10 \%$ of the central value was considered. The Beta distribution was used for parameters constrained in the range $(0-1)$ such as probabilities and utilities. The Gamma distribution was used for all other parameters. The parameters of regression functions predicting the time to events were varied based on their variance-covariance matrix, using the Cholesky decomposition method.

\section{RESULTS}

The base case simulation was carried out for a cohort of 5,000 patients. Such a number ensured the convergence of the probabilistic

\begin{tabular}{lccc}
\hline & Empagliflozin + SoC & SoC & Increments \\
\hline Life Years & 13.8 & 11.8 & 2.0 \\
(undiscounted) & 8.6 & 7.6 & 1.0 \\
QALYs & 15,679 & 10,971 & 4,708 \\
- Drua costs $(€)$ & 9,460 & 3,951 & 5,509 \\
- Event costs $(€)$ & 6,219 & 7,021 & -802 \\
ICER (€/QALY) & & & 4,811 \\
\hline
\end{tabular}

Table IV. Undiscounted (survival) and discounted (QALY and costs) results of the cost-effectiveness analysis (base case)

ICER = incremental cost-effectiveness ratio; QALY = quality-adjusted life year; SoC $=$ standard of care

model and the repeatability of the results. Patients in the treatment arm with empagliflozin as an add-on to the SoC (condition not reimbursed according to the current therapeutic plan) lived for an average of 13.8 years, compared to the 11.8 of the patients in the standard treatment, with a mean life expectancy gain of 2.0 years (Table IV).

The increased survival is related to the lower incidence of cardiovascular and renal events, particularly cardiovascular mortality. Due to the combined effect of improved survival and lower incidence of events, empagliflozin provides patients with a mean increase of 1.0 QALY (discounted, and corresponding to 24 months of life years gained), given by the difference between 8.6 QALYs and 7.6 QALYs 


\begin{tabular}{lcc}
\hline & \multicolumn{2}{c}{ Increments (empagliflozin + SoC vs SoC) } \\
\cline { 2 - 3 } & Base case & Scenario $^{1}$ \\
\hline Life Years & 2.0 & 2.0 \\
QALYS & 1.0 & 1.0 \\
Costs (€) & 4,708 & 2,162 \\
ICER (€/QALY) & 4,811 & 2,205 \\
\hline
\end{tabular}

Table V. Results of the cost-effectiveness analysis in the exploratory scenario ICER: Incremental Cost-Effectiveness Ratio, QALY = quality-adjusted life years; SoC: standard of care;

${ }^{1}$ Pharmaceutical cost in the empagliflozin + SoC arm was calculated by assuming the replacement of other antidiabetic agents, with exception of metformin and insulin

gained in the empagliflozin $+\mathrm{SoC}$ and in the SoC arm, respectively. Cardiovascular and renal costs were averagely lower by $€ 802$, despite the increase in survival. This reduction partially compensates the increase in pharmaceutical costs due to the addition of empagliflozin ( $€ 5,509)$, resulting in a net increase in total cost of $€ 4,708$ ( $€ 15,679$ and $€$ 10,971 for empagliflozin + SoC and the SoC, respectively). Empagliflozin is cost-effective compared to the SoC in patients with T2D and established cardiovascular disease, with a resulting incremental cost-effectiveness ratio (ICER) of 4,811 €/QALY (Table IV). This value is clearly below the cost-effectiveness thresholds commonly considered acceptable (30-50,000 €/QALY).

In the exploratory scenario, it was assumed that the use of empagliflozin occurs through the replacement of other antidiabetic drugs according to the reimbursability limits of the current therapeutic plan (no longer as an addon to basic therapy, as in the baseline scenario), with a reduction of the pharmaceutical

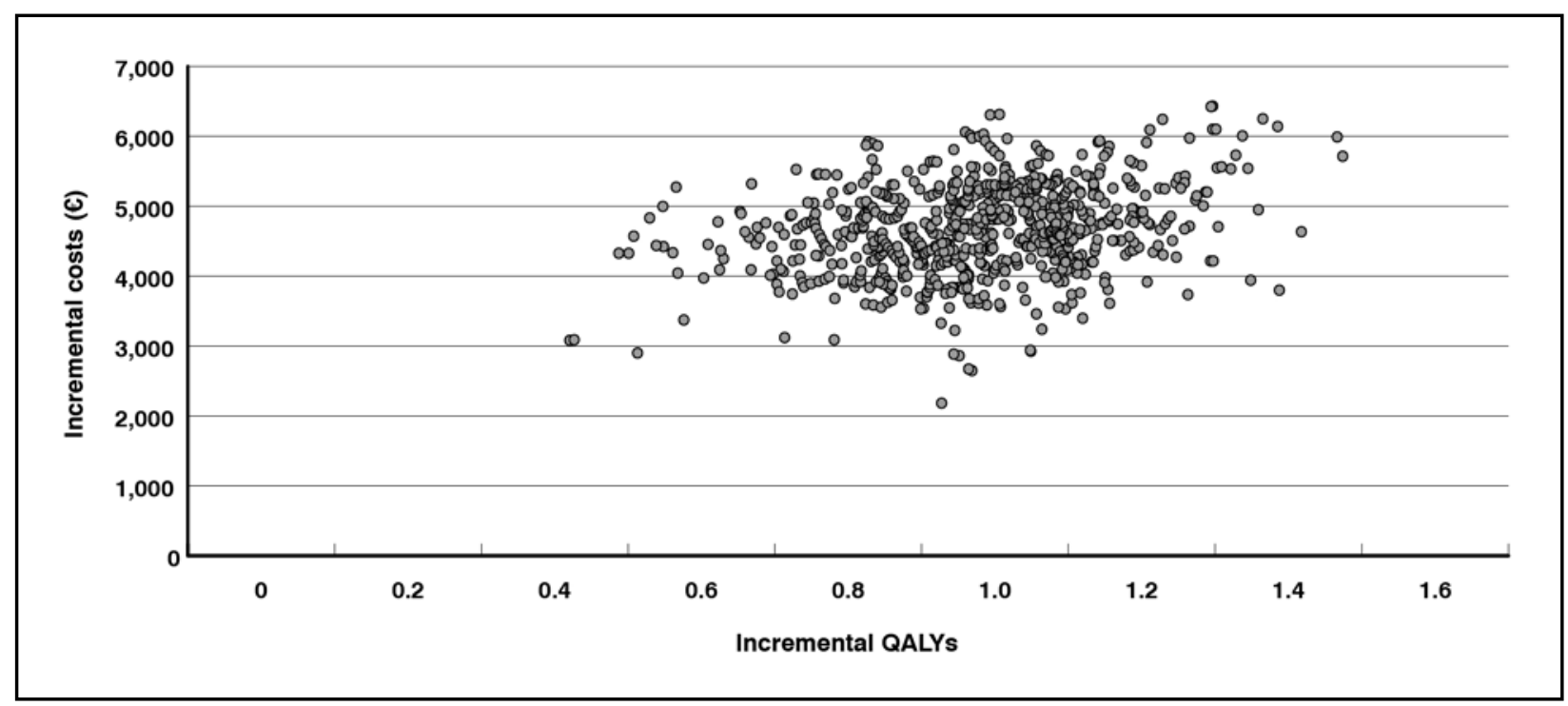

Figure 2. Results of the probabilistic sensitivity analysis (500 iterations)

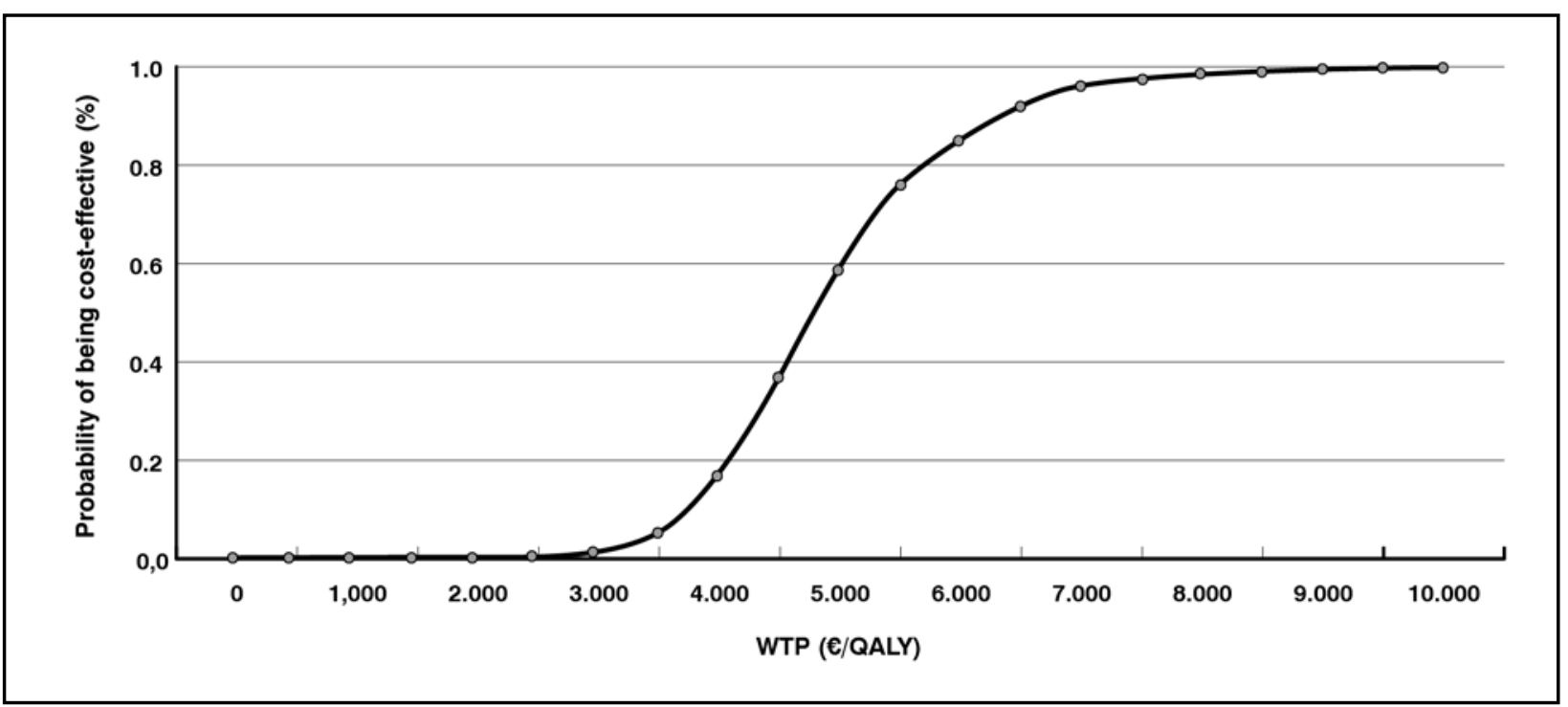

Figure 3. Cost-effectiveness acceptability curve (CEAC)

WTP $=$ cost-effective thresholds (willingness to pay) 
cost for patients treated with empagliflozin + SoC. This reduction led to a total cost difference between the two comparator's arms of $€$ 2,162 , lowering the ICER to $€ 2,205 /$ QALY (Table V).

\section{Sensitivity analysis}

The probabilistic sensitivity analysis allows to simultaneously analyze the impact of various sources of uncertainty. The analysis was performed by assigning a statistic probability distribution to each parameter of the model, so that - at each iteration - the set of input values was slightly different (thus representing the uncertainty on the parameters). The calculation of the results was repeated for 500 iterations. The results of the PSA were graphically analyzed on the cost-effectiveness plane and as cost-effectiveness acceptability curve (CEAC). In the graph of the 500 iterations of the cost-effectiveness plane, the point cloud is sufficiently compact around the central value, demonstrating a good stability of the model data (Figure 2).

The mean value of the ICER resulting from the sensitivity analysis (4,791 €/QALY) is close to the point value of the base case. The ICER credibility range - defined as the range in which $95 \%$ of the analysis values fall - is $3,148-6,503 € /$ QALY. The CEAC is a graphic representation of the likelihood that a treatment is cost-effective as a function of the threshold value for the cost-effectiveness acceptability. The CEAC of the comparison empagliflozin + SoC vs SoC shows that empagliflozin has about a $100 \%$ probability of being cost-effective at acceptability thresholds around 10,000 €/QALY, well below the conventionally considered values (30-50,000 $€ / Q A L Y$ - Figure 3).

\section{DISCUSSION}

Clinical literature has unequivocally shown over the years how T2D can be identified as an important risk factor for cardiovascular events and how the presence of a cardiovascular disease may extend the impact of T2D beyond the mere glycemic control issue. To this regard, the EMPA-REG OUTCOME study highlighted the possibility of choosing therapeutic strategies that can optimize the outcomes of these two intimately related chronic situations. The recent results of the LEADER study with liraglutide and of the SUSTAIN-6 study with semaglutide go in the same direction [24,25]. It, therefore, appears important - for the purpose of a complete technology assessment - to explore in depth the relevant aspects of economic effectiveness and sustainability. The first step in this direction was made by a previous work, through which the same authors of this article investigated the financial sustainability of the adoption of empagliflozin in Italian patients with T2D and established cardiovascular disease [6]. The conclusions reached by the budget impact analysis methodology were that empagliflozin can be a viable therapeutic option, both clinically and economically, and that the hypothesized use of the same treatment could take place without a negative budget impact borne by the Italian National Health Service [6]. The aim of this study was to complete the path with the cost-effectiveness assessment of the treatment in the same setting. The analysis was conducted using a patient-level simulation model, directly based on the EMPA-REG OUTCOME data. The advantage of this type of model is that it allows to extrapolate the risk functions of cardiovascular and renal events directly from the trial data, without the need of surrogate outcomes to estimate results and costs over the long term. On the other hand, the tight relation with the randomized trial constitutes the main limitation of the model itself. As in all randomized clinical trials, in the EMPAREG OUTCOME study, the enrollment was governed by the criteria pre-arranged in the protocol. In the opinion of the authors of this article, this led to the probable exclusion of a series of too complex or too frail patients who may represent a large component of the Italian clinical reality. This limitation is typical of randomized clinical trials, which measure the effects of treatments in an experimental setting, often different from clinical reality. Another common problem related to the use of efficacy data from clinical trials into cost-effectiveness models is related to the extrapolation to the long-term frame. In our case, the follow-up period of the EMPAREG OUTCOME study was relatively long ( 3 years) but in any case, the extrapolation to a lifetime horizon is introducing some uncertainty. This was, however, partially addressed in the probabilistic sensitivity analysis where all parameters of the risk equations were varied within their uncertainty ranges. A second major limitation of the study is linked to the use of utilities for the estimation of the quality of life which are not derived from an Italian study. This limit is, according to the personal experience of the Authors, unfortunately quite common in cost-effectiveness analyses in the Italian setting, due to the poor production of original quality of life studies. Another limitation of the analysis is inherently linked to the use of cost data available in the literature and, in some cases, based on DRG rates, instead of real costs. The results 
of the study showed that patients treated with empagliflozin lived on average two years more than patients with standard treatment, due to the lower incidence of cardiovascular events and mortality. The higher cost, due to the addition of empagliflozin, seems fairly compensated by the benefit on the outcomes, with an ICER of 4,811 €/QALY, a value which is significantly lower than the costeffectiveness thresholds most commonly considered acceptable (30-50,000 €/QALY). Also in the sensitivity analysis, which takes into account the uncertainty of all the input data of the study, the upper limit of the $95 \%$ confidence interval $(3,148-6,503 € / \mathrm{QALY})$ was far from the thresholds, thus showing the reliability of the conclusions produced by the model. It could be interesting to note that the ICER obtained in this study is generally lower than those reported in other important therapeutic areas in Italy. Consider, for example, the cost-effectiveness analysis of a biological product for the treatment of rheumatoid arthritis (ICER $=17,100 € /$ QALY [26]), or that of a tyrosine-kinase inhibitor (TKI) authorized for the treatment of chronic myeloid leukemia (ICER = 22,529 €/QALY [27]). It is, therefore, reasonable to consider reassuring the cost-effectiveness results of empagliflozin in the treatment of subjects with T2D and established cardiovascular disease, even within the discussed limitations of the study. Empagliflozin, therefore, represents an efficient investment in terms of allocation of the Italian NHS's resources, since it ensures a lower additional cost per QALY than other biotechnological treatments. All this considered, and in concomitance with the results of the budget impact analysis [6], we can, therefore, conclude that empagliflozin can be a valid therapeutic option in subjects with $\mathrm{T} 2 \mathrm{D}$ and established cardiovascular disease, both from a clinical and economic perspective.

\section{Conflicts of interest}

SI reports grants from Boehringer Ingelheim, during the conduct of the study.

EM reports personal fees from Fondazione Charta, during the conduct of the study; grants and personal fees from Boehringer Ingelheim, AstraZeneca, Janssen, Eli Lilly, and Novo Nordisk, and personal fees from Sanofi and Takeda, outside the present work.

OR reports personal fees (salary for employment) from Evidera Inc., during the conduct of the study APM reports personal fees from Novartis,

Bayer, Cardiorentis, and Fresenius, outside the present work

\section{APPENDIX A}

Time dependent parametric survival analyses of the EMPA-REG OUTCOME trial data were conducted to characterize clinical event rates over time under standard of care alone and with empagliflozin in addition to the standard of care therapy (Table IA).

\section{Steps for determining predictors and parametric distribution}

A systematic two-stage analytical process was used to develop event-free survival functions for the clinical events included in the model.

1. Population-level time-to-event survival curves were fit to the EMPA-REG OUTCOME trial data considering various potential statistical distributions. Empirical time-to-event curves were derived using the Kaplan-Meier method to ascertain the shape of the distribution in each treatment arm. Extrapolation of time-to-event distributions beyond the trial timeframe was handled with parametric survival analyses methods. Statistical distributions (i.e., exponential, Weibull, log-Normal, log-Logistic, Gompertz) that can account for the typically skewed distributions of time-to-event variables were considered in the fitting process. The process of selecting the best fitting distribution involved both statistical and clinical considerations, including goodness of fit (Akaike's Information Criteria and Bayesian Information Criterion), plausibility of results, and parsimony (preference for the simplest functional form). The approach to fitting the curves was as outlined in Ishak et al. [28], which provides the technical details behind the steps of the analyses. Results of this analysis provide the parameters (i.e., shape, scale) for the baseline distribution of each KM curve for each arm (base overall curve). Survival analyses were performed in SAS version 9.4.

2. A Cox proportional-hazards analysis was conducted to develop individual patient-level risk equations based on patient characteristics and event history. Baseline and time-dependent predictors that impact the risk of the outcome event were tested for significance in the risk equations. Candidate characteristics for predictors were selected based on clinical relevance as indicated in prespecified analysis for the trial endpoints, and included treatment arm (pooled empagliflozin doses versus placebo), demographic information (age, sex, geographic region), baseline biomarkers (HbAlc, BMI, eGFR), baseline event history (cardiovascular, cerebrovascular, and peripheral arterial disease), and cardiovascular and renal events experienced during trial 


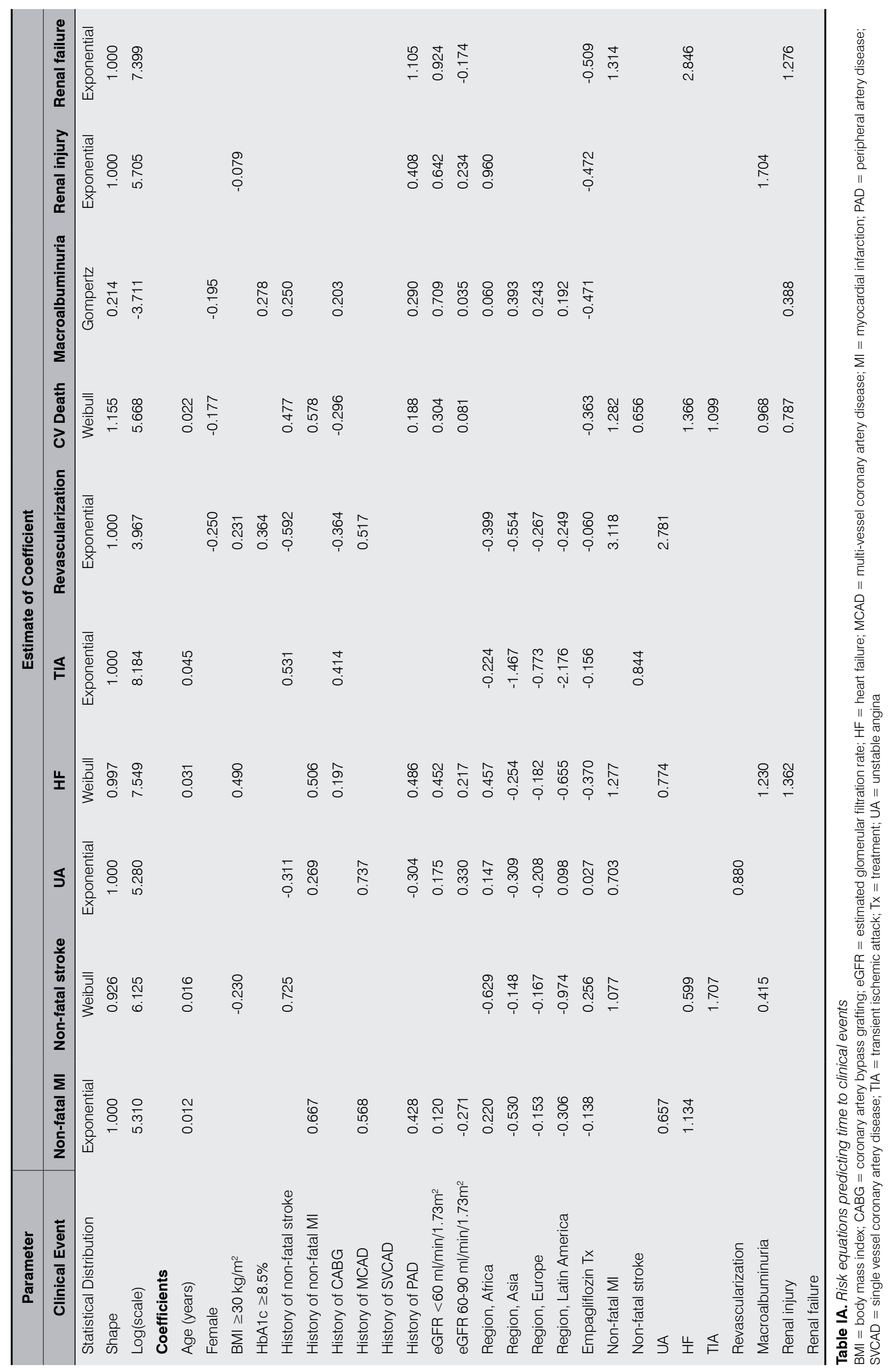


follow-up. The predictor selection strategy involved first assessing each potential predictor for significance $(\mathrm{p}<0.2)$ in univariate analysis to determine whether it is associated with the outcome on its own. The significant predictors were combined in multivariate parametric equations, trimmed one variable at a time (in order of highest $\mathrm{p}$-value) to exclude variables not significant at $p<0.2$. The final mul- tivariate equations contain predictors that have a significant effect or important prognostic factors that show a nonnegligible effect size. The Cox regression analysis provided tables of hazard ratios for covariates included in each equation that are used to adjust the base curves. Time-dependent multivariate parametric survival regression models were built using $\mathrm{R}$ version 3.2.2.

\section{REFERENCES}

1. Sarwar N, Gao P, Seshasai SR, et al. Diabetes mellitus, fasting blood glucose concentration, and risk of vascular disease: a collaborative meta-analysis of 102 prospective studies. Lancet 2010; 375: 2215-22; https://doi.org/10.1016/ S0140-6736(10)60484-9

2. Beckman JA, Creager MA, Libby P. Diabetes and atherosclerosis: epidemiology, pathophysiology, and management. JAMA 2002; 287: 2570-81; https://doi.org/10.1001/jama.287.19.2570

3. World Heart Federation. Diabetes. Available at: http:/www.world-heart-federation.org/cardiovascular-health/ cardiovascular-disease-risk-factors/diabetes/ (last accessed May 2017)

4. Zinman B, Wanner C, Lachin JM, et al.; EMPA-REG OUTCOME Investigators. Empagliflozin, Cardiovascular Outcomes, and Mortality in Type 2 Diabetes. NEngl J Med 2015; 373: 2117-28; https://doi.org/10.1056/NEJMoa1504720

5. Wanner C, Inzucchi SE, Lachin JM, et al.; EMPA-REG OUTCOME Investigators. Empagliflozin and Progression of Kidney Disease in Type 2 Diabetes. N Engl J Med 2016; 375: 323-34; https://doi.org/10.1056/NEJMoa1515920

6. Iannazzo S, Maggioni AP, Mannucci E. Analisi di impatto sul budget di empagliflozin nel trattamento dei pazienti con diabete di tipo 2 e malattia cardiovascolare accertata. GRHTA 2017; 4: e122-32

7. Kansal A, Zheng Y, Proskorovsky I, et al. Long-term Economic Outcomes of Empagliflozin (Jardiance) Treatment in Type 2 Diabetes Mellitus (T2DM) based on the EMPA-REG OUTCOME Trial. American Diabetes Association 76th Scientific Sessions, June 10-14, 2016. New Orleans, LA, USA

8. Gibbons I, Kandaswamy P, Tebboth A, et al. Cost-effectiveness of empagliflozin (Jardiance) in the treatment of patients with Type 2 Diabetes Mellitus (T2DM) in the UK based on EMPA-REG OUTCOME data. ISPOR 19th Annual European Congress. October 2016, Vienna, Austria

9. Wanner C, Inzucchi SE, Lachin JM, et al.; EMPA-REG OUTCOME Investigators. Empagliflozin and Progression of Kidney Disease in Type 2 Diabetes. N Engl J Med 2016; 375: 323-34; https://doi.org/10.1056/NEJMoa1515920

10. ISTAT. Tavole di mortalità della popolazione residente. Anno: 2015. Available at: http://demo.istat.it (last accessed March 2017)

11. Lombardo F, Spila Alegiani S, Maggini M, et al., on behalf of Studio DAI. Istituto Superiore di Sanità. Prevalenza e incidenza delle complicanze del diabete: studio DAI. Rapporti ISTISAN 07/25, 2017

12. AMD Associazione Medici Diabetologi. Annali AMD 2012

13. Szende A, Janssen B, Cabases J (Eds). Self-Reported Population Health: An International Perspective based on EQ-5D. Springer, 2014

14. Sullivan PW, Slejko JF, Sculpher MJ, et al. Catalogue of EQ-5D scores for the United Kingdom. Med Decis Making 2011; 31: 800-4; https://doi.org/10.1177/0272989X11401031

15. Sullivan PW, Ghushchyan VH. EQ-5D Scores for Diabetes-Related Comorbidities. Value Health 2016; 19: 1002-8; https://doi.org/10.1016/j.jval.2016.05.018

16. Lindgren P, Graff J, Olsson AG, et al. Cost-effectiveness of high-dose atorvastatin compared with regular dose simvastatin. Eur Heart J 2007; 28: 1448-53; https://doi.org/10.1093/eurheartj/ehm020

17. QuintilesIMS, Patient Analyzer MAT Marzo 2016. Data on file

18. CODIFA - L'Informatore Farmaceutico. Available at: http://www.codifa.it/ (last accessed May 2017)

19. Agenzia Italiana del Farmaco (AIFA). Lista di trasparenza degli equivalenti aggiornata ad aprile 2016. Available at: http://www.agenziafarmaco.gov.it/it/content/liste-di-trasparenza-e-rimborsabilit\%C3\%A0 (last accessed May 2017) 
20. QuintilesIMS. Dati di mercato Sell-in SU FisYr/12/2015 (Absolute). Data on file

21. Ministero della Salute. DECRETO 18 ottobre 2012. Remunerazione prestazioni di assistenza ospedaliera per acuti, assistenza ospedaliera di riabilitazione e di lungodegenza post acuzie e di assistenza specialistica ambulatoriale. GU n.23 del 28-1-2013 - Suppl. Ordinario n. 8

22. Ministero della Salute. Ricoveri ospedalieri (SDO). Dati 2014. Available at: http://www.salute.gov.it/portale/temi/ p2_4.jsp?area=ricoveriOspedalieri (last accessed April 2017)

23. Censis (Centro Studi Investimenti Sociali) - SIN (Società Italiana di Nefrologia) - Centro Nazionale Trapianti. Il valore del trapianto - Sintesi dei Risultati, 17 dicembre 2013. Available at: http://www.censis.it (last accessed March 2017)

24. Marso SP, Daniels GH, Brown-Frandsen K, et al.; LEADER Steering Committee.; LEADER Trial Investigators. Liraglutide and Cardiovascular Outcomes in Type 2 Diabetes. $N$ Engl J Med 2016; 375: 311-22; https://doi. org/10.1056/NEJMoa1603827

25. Marso SP, Bain SC, Consoli A, et al.; SUSTAIN-6 Investigators. Semaglutide and Cardiovascular Outcomes in Patients with Type 2 Diabetes. N Engl J Med 2016; 375: 1834-1844; https://doi.org/10.1056/NEJMoa1607141

26. Diamantopoulos A, Benucci M, Capri S, et al. Economic evaluation of tocilizumab combination in the treatment of moderate-to-severe rheumatoid arthritis in Italy. J Med Econ 2012; 15: 576-85; https://doi.org/10.3111/136969 98.2012.665110

27. Lucioni C, Iannazzo S, Mazzi S, et al. Valutazione di costo-efficacia di ponatinib nella terapia della leucemia mieloide cronica in Italia. GRHTA 2015; 2: 1-16

28. Ishak KJ, Kreif N, Benedict A, et al. Overview of parametric survival analysis for health-economic applications. Pharmacoeconomics 2013; 31: 663-75; https://doi.org/10.1007/s40273-013-0064-3 